This article is licensed under the Creative Commons Attribution-NonCommercial 4.0 International License (CC BY-NC) (http://www.karger.com/Services/OpenAccessLicense). Usage and distribution for commercial purposes requires written permission.

\title{
Enoxaparin-Induced Liver Injury
}

\author{
Shehriyar Mehershahi ${ }^{a, b} \quad$ Nikhitha Mantri $^{b} \quad$ Aneesh Kumar ${ }^{b}$ \\ Shaikh Danial ${ }^{a, b}$ Patel Harish ${ }^{a, b}$ \\ aDepartment of Gastroenterology, Bronx Care Health System, Bronx, NY, USA; \\ ${ }^{b}$ Department of Internal Medicine, Bronx Care Health System, Bronx, NY, USA
}

\section{Keywords}

Enoxaparin · Drug-induced liver injury · Viral hepatitis · Non-alcoholic fatty liver disease

\begin{abstract}
Enoxaparin, a form of low-molecular-weight heparin, can cause a rare, underreported, and often reversible form of hepatocellular injury. This report describes a case of enoxaparin-induced hepatotoxicity in a 61-year-old male diagnosed with pulmonary embolism. Elevations of liver enzymes were noted within 1 week of starting the drug, followed by a dramatic improvement upon its discontinuation, with subsequent normalization in the following days.

\footnotetext{
(C) 2020 The Author(s)

Published by S. Karger AG, Basel
}

\section{Introduction}

Anticoagulants are commonly used for the prevention and treatment of various thromboembolic diseases. Enoxaparin is a low-molecular-weight heparin (LMWH) that acts by binding to antithrombin and irreversibly inactivates clotting factor Xa. It is smaller in size and more uniform compared to unfractionated heparin, and thereby demonstrates a greater affinity for 
factor Xa [1]. LMWH has a superior safety profile compared to that of unfractionated heparin and is thus preferred over the latter. Bleeding and thrombocytopenia are common side effects, whereas only a few reports of enoxaparin-induced liver injury (EILI) exist [2]. The Food and Drug Administration's (FDA) Adverse Event Reporting System (FAERS) reports hepatic toxicity as an adverse event in only $4 \%$ of all enoxaparin-related adverse events [3]. Herein, we present a case of a male with pulmonary embolism, who developed transaminitis when treated with enoxaparin.

\section{Case Presentation}

A 61-year-old male presented to our emergency department complaining of worsening shortness of breath associated with pleuritic chest pain for 3 days. His medical comorbidities included hypertension, type 2 diabetes mellitus, asthma, seizure disorder, obstructive sleep apnea, and a history of pulmonary embolism. His only surgery was an inguinal hernia repair in the past. He reported an allergy to angiotensin-converting enzyme inhibitors. He denied alcohol consumption, smoking, or recreational drug use. He had not travelled outside the United States, nor did he encounter any sick contacts. He denied the use of any herbal preparations or over-the-counter medications. Coumadin was the only prescription medication he was taking on a regular basis.

On examination, the patient was a well-built man in respiratory distress. He was tachypneic and hypoxic, saturating at $88 \%\left(\mathrm{SpO}_{2}\right)$ on room air. Blood pressure was $130 / 80$, heart rate $88 \mathrm{bpm}$, and he was afebrile at $97^{\circ} \mathrm{F}$. On inspection, no icterus, lymphadenopathy, or peripheral edema was noted. On auscultation, his lungs were clear bilaterally, and no heart murmurs were appreciated. His abdomen was soft, non-tender with no hepatosplenomegaly, and bowel sounds were normal.

Initial troponin and pro-brain natriuretic peptide levels were elevated. A computerized tomography with contrast of the chest revealed multiple filling defects within the pulmonary arterial system, indicative of bilateral acute on chronic thromboembolic disease. The patient was started on enoxaparin $120 \mathrm{mg}$ twice a day in addition to his coumadin, as an aim to bridge to a therapeutic international normalized ratio between 2 and 3 .

On admission, his liver enzymes were within normal limits as per our institute laboratory values, with an alanine transaminase (ALT) of 28 units/L, aspartate transaminase (AST) of 13 units/L, alkaline phosphatase (ALP) of 95 units/L, albumin of $3.4 \mathrm{~g} / \mathrm{dL}$, total bilirubin of 0.4 $\mathrm{mg} / \mathrm{dL}$, and conjugated bilirubin of $0.1 \mathrm{mg} / \mathrm{dL}$. They remained as such, up until day 6 , when a slight elevation was noted. On day 6, the AST was 72 units/L, ALT was 84 units/L, and ALP was 161 units/L. An R ratio, the initial step of the Roussel Uclaf Causality Assessment Method (RUCAM), was calculated to be 2.64, consistent with a mixed (hepatocellular and cholestatic) pattern of liver injury. At this point, no changes were made to the patient's treatment regimen. On day 7, his liver enzymes peaked with AST of 143 units/L, ALT of 231 units/L, and ALP of 123 units/L as shown in Table 1. R ratio from these laboratory values was 5.56, consistent with hepatocellular pattern of liver injury.

An ultrasound and computerized tomography of the abdomen was negative for any significant pathology. Echocardiogram of the heart revealed a normal ejection fraction and normal diastolic function. Serologies for hepatitis A, hepatitis B, hepatitis C, Epstein Barr virus, 


\section{Case Reports in Gastroenterology}

Case Rep Gastroenterol 2020;14:315-319

DOI: 10.1159/000508471

(c) 2020 The Author(s). Published by S. Karger AG, Base www.karger.com/crg

Mehershahi et al.: Enoxaparin-Induced Liver Injury

and cytomegalovirus were all negative. Antinuclear antibody screen, anti-mitochondrial antibody, anti-smooth muscle antibody, and anti-liver kidney microsomal antibody were also negative. Serum alpha- 1 antitrypsin and serum ceruloplasmin levels were normal.

On day 7 of admission, enoxaparin was discontinued secondary to a therapeutic international normalized ratio. His liver function tests demonstrated a dramatic and steady decline thereafter. He was subsequently discharged on coumadin, and a 2-month follow-up revealed complete normalization of his liver enzymes.

\section{Discussion}

Reports of anticoagulant-induced liver injury first appeared 40 years ago [3]. EILI is uncommon and reversible [2]. The mechanism of transaminitis caused by heparin and heparin products is unknown, although various hypotheses have been suggested. Dukes et al. [4] suggested that the cellular damage could be cause by a direct toxic effect or due to hypersensitivity reactions. A case series of EILI demonstrated balloon degeneration and multiple foci of hepatocyte necrosis on liver biopsy of one of the subjects. Complement-mediated hepatocellular injury was thought to be the underlying pathology [5]. Clinical trials and prospective studies have reported hepatotoxicity secondary to LMWH with a frequency between 5 and 9\%, where hepatotoxicity is considered as AST and ALT elevations more than 3 times the upper limit of normal [2].

The elevation in liver enzymes is usually seen after 5-8 days of initiation of heparin products and normalizes or improves within 2 weeks of cessation of the drug [6]. Our case describes a male patient with normal liver function tests, which was started on enoxaparin and developed transaminitis 6 days after. The transaminitis reached its peak on day 9. All other known causes of liver injury were tested for and excluded. Following discontinuation of enoxaparin, liver enzymes began to improve and normalized within 8 weeks. Most patients with EILI are asymptomatic; however, symptoms such as nausea, vomiting, and abdominal pain may manifest [7]. Our patient did not exhibit any of the aforementioned symptoms.

As per the Naranjo scale [8], there is a "probable" cause of liver injury in our case. The calculated $\mathrm{R}$ factor was $>5$, suggesting a hepatocellular pattern of injury, rather than a cholestatic one [9]. At the peak of liver injury, the RUCAM score [10] was 9, suggesting a high probable likelihood that enoxaparin was the causative agent.

\section{Conclusion}

Enoxaparin's impact on the liver is seldom considered by clinicians when prescribing anticoagulants. Its benign, self-limiting, and reversible effects may not warrant extensive investigations; however, it is imperative to know that higher doses and a longer duration of treatment is associated with higher probability of liver injury [3]. Further research is needed to establish the mechanism of injury and how to prevent it. The benefits of obtaining baseline LFTs prior to initiating enoxaparin therapy also remain unclear. However, increased awareness regarding this condition can minimize excessive and unnecessary investigations, patient anxiety, and healthcare costs. 


\section{Case Reports in Gastroenterology}

Case Rep Gastroenterol 2020;14:315-319

DOI: $10.1159 / 00050847$

(C) 2020 The Author(s). Published by S. Karger AG, Base www.karger.com/crg

Mehershahi et al.: Enoxaparin-Induced Liver Injury

\section{Statement of Ethics}

The patient provided written informed consent.

\section{Disclosure Statement}

The authors declare that they have no conflicts of interest to disclose.

\section{Funding Sources}

The authors did not receive any funding.

\section{Author Contributions}

All authors certify that he or she has participated sufficiently in the intellectual content, the analysis of data. Each author has reviewed the final version of the manuscript and approves it for publication. Should the editors request the data upon which the work is based, the authors shall produce it.

Shehriyar Mehershahi: preparation of manuscript and literature analysis/search.

Nikhitha Mantri: preparation of manuscript and literature analysis/search.

Aneesh Kumar: preparation of case presentation.

Shaikh Danial: literature analysis/search.

Patel Harish: preparation of manuscript.

\section{References}

1 Hirsh J, Levine MN. Low molecular weight heparin. Blood. 1992 Jan;79(1):1-17.

2 Carlson MK, Gleason PP, Sen S. Elevation of hepatic transaminases after enoxaparin use: case report and review of unfractionated and low-molecular-weight heparin-induced hepatotoxicity. Pharmacotherapy. 2001 Jan;21(1):108-13.

3 Hahn KJ, Morales SJ, Lewis JH. Enoxaparin-Induced Liver Injury: Case Report and Review of the Literature and FDA Adverse Event Reporting System (FAERS). Drug Saf Case Rep. 2015 Dec;2(1):17.

4 Dukes GE Jr, Sanders SW, Russo J Jr, Swenson E, Burnakis TG, Saffle JR, et al. Transaminase elevations in patients receiving bovine or porcine heparin. Ann Intern Med. 1984 May;100(5):646-50.

5 Hui CK, Yuen MF, Ng IO, Tsang KW, Fong GC, Lai CL. Low molecular weight heparin-induced liver toxicity. J Clin Pharmacol. 2001 Jun;41(6):691-4.

6 Arora N, Goldhaber SZ. Anticoagulants and transaminase elevation. Circulation. 2006 Apr;113(15):e698702.

7 Baker EL, Loewenthal T, Salerno E, Baker WL. Probable enoxaparin-induced hepatotoxicity. Am J Heal Pharm; 2009. https://doi.org/10.2146/ajhp080311.

8 Naranjo CA, Busto U, Sellers EM, Sandor P, Ruiz I, Roberts EA, et al. A method for estimating the probability of adverse drug reactions. Clin Pharmacol Ther. 1981 Aug;30(2):239-45.

9 Danan G, Teschke R. RUCAM in drug and herb induced liver injury: the update. Int J Mol Sci. 2015 Dec;17(1):E14. 


\section{Case Reports in Gastroenterology}

Case Rep Gastroenterol 2020;14:315-319

Mehershahi et al.: Enoxaparin-Induced Liver Injury

10 Danan G, Teschke R. Drug-Induced Liver Injury: Why is the Roussel Uclaf Causality Assessment Method (RUCAM) Still Used 25 Years After Its Launch? Drug Saf. 2018 Aug;41(8):735-43.

Table 1. Laboratory parameters over the course of hospitalization

\begin{tabular}{|c|c|c|c|c|c|c|c|c|c|c|}
\hline \multirow[t]{2}{*}{ Laboratory parameter } & \multirow[t]{2}{*}{ Normal value } & \multicolumn{9}{|c|}{ Day of hospitalization } \\
\hline & & $1^{\mathrm{a}}$ & 5 & 6 & $7 \mathrm{a}$ & 8 & 9 & 10 & 13 & 63 \\
\hline AST, unit/L & $9-48$ & 27 & 17 & 72 & 143 & 110 & 89 & 76 & 33 & 17 \\
\hline ALT, unit/L & $5-40$ & 23 & 30 & 84 & 231 & 215 & 199 & 185 & 118 & 30 \\
\hline ALP, unit/L & $56-155$ & 126 & 102 & 123 & 161 & 163 & 173 & 166 & 146 & 97 \\
\hline INR & $0.90-1.09$ & 1.26 & 1.25 & 1.79 & 2.08 & 2.43 & 2.09 & 2.15 & 2.17 & 2.43 \\
\hline Total bilirubin, mg/dL & $0.2-1.1$ & 0.6 & 0.2 & 0.4 & 0.3 & 0.3 & 0.3 & 0.3 & 0.4 & 0.4 \\
\hline Conjugated bilirubin, $\mathrm{mg} / \mathrm{dL}$ & $0.0-0.3$ & 0.2 & 0.2 & 0.1 & 0.1 & 0.1 & 0.1 & 0.1 & 0.1 & 0.2 \\
\hline R ratio ${ }^{b}$ & & & & 2.64 & & & 5.56 & & & \\
\hline RUCAM score $^{c}$ & & & & 8 & & & 9 & & & \\
\hline
\end{tabular}

ALP, alkaline phosphatase; ALT, alanine transaminase; AST, aspartate transaminase; INR, international normalized ratio; RUCAM, Roussel Uclaf Causality Assessment Method; ULN, upper limit of normal. a Enoxaparin was initiated on day 1 and continued until day 7. ${ }^{\mathrm{b}} \mathrm{R}$ ratio: (ALT value $\div$ ALT ULN $) \div$ (Alk P value $\div$ Alk P ULN). R ratios of $>5$ : hepatocellular injury, <2: cholestatic injury, 2-5: mixed pattern of injury. 'RUCAM score: type of liver injury: hepatocellular; time of onset of the event: first exposure; time from drug intake until reaction onset: $<5-$ $>90$ days ( +1 points); time from drug withdrawal until reaction onset: $\leq 15$ days ( +1 point); alcohol risk factor: absent ( 0 points); age risk factor: $<55$ years ( 0 points); course of reaction: $>50 \%$ improvement 8 days $(+3$ points); concomitant therapy: time to onset compatible but known reaction ( +2 points); exclusion of nondrug-related causes: ruled out ( +2 points); previous information: reaction labeled in the product's characteristics ( +2 points); response to re-administration: positive $(+3$ points). Bold values indicate abnormal values. 